\title{
Corticosteroids as adjunctive therapy with antibiotics in the treatment of children with septic arthritis: a meta-analysis
}

This article was published in the following Dove Press journal: Drug Design, Development and Therapy

\section{Ya-fei Qin \\ Zhi-jun Li \\ Hui Li}

Department of Orthopedics, Tianjin Medical University General Hospital, Tianjin 300052, China
Correspondence: Hui Li

Department of Orthopedics, Tianjin Medical University General Hospital, No. 154 Anshan Road, Heping District, Tianjin 300052, China

Tel +86 I39 2055101 I

Fax +8602260362062

Email lihuitjmu@।63.com
Purpose: We performed a meta-analysis to systematically assess the effect of adjunctive administration of dexamethasone with antibiotic therapy in the clinical course of septic arthritis (SA) in children.

Method: Potential academic articles were identified from the Cochrane Library, Medline, PubMed, Embase, ScienceDirect, and other databases. The time range we retrieved from was from the inception of electronic databases to January 2018. The reference lists of identified studies were manually checked to identify other potentially eligible trials. The STATA version 11.0 (Stata Corporation, College Station, TX, USA) was used to analyze the pooled data.

Results: Three randomized controlled trials, and one retrospective cohort study were included in the meta-analysis. There were significant differences in the days of hospitalization (mean difference $[\mathrm{MD}]=-4.226,95 \% \mathrm{CI}:-4.785$ to $-3.667, P=0.001$ ), the days of intravenous antibiotics treatment $(\mathrm{MD}=-3.593,95 \% \mathrm{CI}:-4.825$ to $-2.361, P=0.001)$, the days of oral antibiotics treatment $(\mathrm{MD}=-1.658,95 \% \mathrm{CI}:-2.539$ to $-0.777, P=0.001)$, and the days to normalization of C-reactive protein $(\mathrm{MD}=-3.075,95 \% \mathrm{CI}:-3.362$ to $-2.788, P=0.001)$.

Conclusion: The present meta-analysis base points strongly toward a beneficial effect for corticosteroids in SA. Corticosteroids as adjunctive therapy with antibiotics in the treatment of children with SA could shorten the number of days of hospitalization, the days of intravenous antibiotics treatment, the days of oral antibiotics treatment, and the days to normalization of C-reactive protein. We recommend corticosteroids as adjunctive therapy with antibiotics in the treatment of children with SA.

Keywords: glucocorticoids, child, septic arthritis, meta-analysis

\section{Introduction}

Septic arthritis (SA) is an acute infectious disease of the skeletal system caused by pyogenic bacteria. Acute SA is a potentially devastating disease in childhood that is responsible for significant morbidity and even mortality $(1 \%-15 \%) .{ }^{1}$ The higher incidence of infection in those of a younger age carries the great concerns with this issue. Early application of effective antibiotics is the key to the treatment of SA, but there are still 10\%-25\% of children with joint dysfunction, abnormal bone growth, and other sequelae even after the bacteria have been eradicated with antimicrobial treatment. ${ }^{2,3}$

The sequelae of joint infection in children following anti-infective therapy may be related to the abnormal inflammatory response of the host immune system to the pathogen. ${ }^{4}$ These pro-inflammatory cytokines in the joint cavity, such as interleukin (IL)-1, tumor necrosis factor, IL-17, and IL-6 stimulating osteoclast differentiation lead to subsequent 
bone reabsorption and cartilage degradation. ${ }^{5}$ The results of animal experiments show that it is necessary to carry out antiinflammatory treatment to inhibit the inflammatory reaction and reduce the destruction of articular cartilage on the basis of effective antibiotic treatment of SA. ${ }^{6-8}$ Glucocorticoid therapy has been one well-established method of reducing inflammation reaction for decades. ${ }^{9}$ Corticosteroid administration and sequential antibiotic therapy have beneficial effects in mouse models on treatment to inhibit the inflammatory reaction. ${ }^{8}$ These findings were supported by clinical studies in children with SA. The first, by Arti et al, ${ }^{10}$ showed that routine antibiotic therapy and intravenous dexamethasone can reduce the clinical symptoms, accelerate recovery, and restore daily activities in children with SA. The second study was conducted by Odio et $\mathrm{l}^{11}$ in 123 patients using a double-blind, randomized, placebocontrolled study design. Odio et $\mathrm{al}^{11}$ found that a 4-day course of low-dose dexamethasone reduced residual joint dysfunction and obviously shortened the duration of symptoms in children with SA. Although, the benefits of corticosteroids as adjunctive therapy with antibiotics in the treatment of children with SA are encouraging, supplemental glucocorticoid therapy is still a debatable point. ${ }^{12,13}$ For example, chronic use of steroids in children may lead to adrenal insufficiency, growth retardation, and osteoporosis. ${ }^{13}$ Furthermore, treatment with glucocorticoids may hide symptoms of infection. ${ }^{14}$ A recent systematic review confirms that further studies are needed to clarify the results. ${ }^{15}$ Therefore, we necessarily performed a metaanalysis to investigate the effectiveness of adjunctive administration of corticosteroids in the treatment of SA in children.

\section{Materials and methods}

The present study was completed according to the preferred reporting items for systematic review and meta-analyses (PRISMA) statement (Checklist S1).

\section{Literature and search strategy}

The electronic databases, including Cochrane Library, Medline, PubMed, Embase, ScienceDirect, and other databases were retrieved to identify the publications randomized controlled trials (RCTs) and randomized cohort study (RCS) exploring the adjunctive administration of dexamethasone to antibiotic therapy in the clinical course of SA in children from the inception of electronic databases to January 2018. Structured search strategies were used in combination, according to Boolean logic: (steroids OR corticosteroids OR glucocorticoids OR dexamethasone) AND (infectious arthritis OR bacterial arthritis OR SA OR suppurative arthritis). In addition, the research on the appraisal reference list was manually reviewed for other potential trials that should be included. The process was iterated until no further articles could be determined. The meta-analysis was based on acknowledged PRISMA guidelines (the prioritized reported items for systematic review and meta-analysis).

\section{Inclusion and exclusion criteria}

If the article met the following criteria in accordance with PICOS, the article was considered to be included in the current meta-analysis: 1) population: children with SA; 2) intervention: corticosteroids as adjunctive therapy with antibiotics; 3 ) comparison intervention: dexamethasone group to placebo group; 4) outcome measures, 1 or more of the following outcomes were reported: the days of hospitalization, the days of intravenous antibiotics treatment, the days of oral antibiotics treatment, and the days to normalization of $\mathrm{C}$-reactive protein (CRP); 5) an official published full-text English-written RCTs or RCS. Exclusion criteria: 1) Non-English language publications, case reports, comments, letters, editorials, protocols, guidelines, and review papers were excluded; 2) animal studies were excluded; 3) articles were also excluded if they are concentrated on non-intravenous application of corticosteroids. A clear description on how the diagnosis of SA is demonstrated in the articles: 1) acute symptom of joint (fever, solitary joint pain, swelling, tenderness, limited range of motion, limp, or afebrile neonate with extremity disuse); 2) elevations of CRP or erythrocyte sedimentation rate; and 3) a turbid purulent appearance of joint fluid and an elevated white blood cell count.

\section{Data extraction and outcome measures}

Two of the reviewers independently extracted data from the included studies. The following essential information was captured: first author name, publication year, sample size, study design, and outcomes. Other relevant data such as patient characteristics and literature quality score were also extracted from individual studies. The extracted data: median, range and size of the trial, and mean difference (MD) and SD were input into the designed standardized table. When there were differences in opinion, another author had the final decision. The outcome measurements were the number of days of hospitalization, both intravenous and oral antibiotics treatment, and the number of days to normalization of CRP. Complications included incidence of infection, fracture, nerve and vascular injury, and thrombosis. All results of the meta-analysis are presented in Table 1.

\section{Quality assessment and statistical analysis}

Tools from Cochrane Bone, the Joint and Muscle Trauma Group and the methodological index for non-randomized 
Table I The results of meta-analysis

\begin{tabular}{|c|c|c|c|c|c|c|c|}
\hline \multirow[t]{2}{*}{ Outcome } & \multirow[t]{2}{*}{ Studies } & \multirow{2}{*}{$\begin{array}{l}\text { Groups } \\
\text { (D/P) }\end{array}$} & \multicolumn{3}{|l|}{ Overall effect } & \multicolumn{2}{|c|}{ Heterogeneity } \\
\hline & & & Effect estimate & $95 \% \mathrm{Cl}$ & $P$-value & ${ }^{22}(\%)$ & $P$-value \\
\hline Days of hospitalization & 2 & $56 / 120$ & -4.226 & -4.785 to -3.667 & 0.000 & 0.0 & 0.524 \\
\hline Days to normalization of CRP & 3 & III/I77 & -3.075 & -3.362 to -2.788 & 0.000 & 0.0 & 0.728 \\
\hline Days of intravenous antibiotics treatment & 3 & $111 / 177$ & -3.593 & -4.825 to -2.361 & 0.000 & 61.8 & 0.073 \\
\hline Days of oral antibiotics treatment & 2 & $85 / 83$ & -1.658 & -2.539 to -0.777 & 0.000 & 5.9 & 0.303 \\
\hline
\end{tabular}

Abbreviations: CRP, C-reactive protein; D, dexamethasone; $P$, placebo.

studies (MINORS) were used to evaluate the quality of the included studies. The literature quality evaluation was conducted separately by two reviewers. Consensus was reached through consultation for divergence. We used STATA version 11.0 (Stata Corporation) for statistical analyses. When $I^{2}>50 \%$, we considered the data to have obvious heterogeneity, and we conducted a meta-analysis using a random-effect model according to the Cochrane Handbook for Systematic Reviews of Interventions (version 5.1.0). Otherwise, the fixed-effect model was performed. The results of the continuous outcomes (the number of days of hospitalization, intravenous and oral antibiotics treatment, and the number of days to normalization of CRP) were expressed as the MD with 95\% CIs. For discontinuous variable outcomes, a risk difference or relative risk with $95 \%$ CIs was applied for the assessment.

\section{Results}

\section{Search results}

The selection process is illustrated in Figure 1, the original database (Cochrane Library, Medline, PubMed, Embase, and ScienceDirect) search yielded 236 records. Of them, 32 articles were eliminated because of duplication. Another 186 articles were excluded for various reasons (unavailable data, case report, reviews, and irrelevant articles). The remaining 18 articles were reviewed in their entity. We excluded

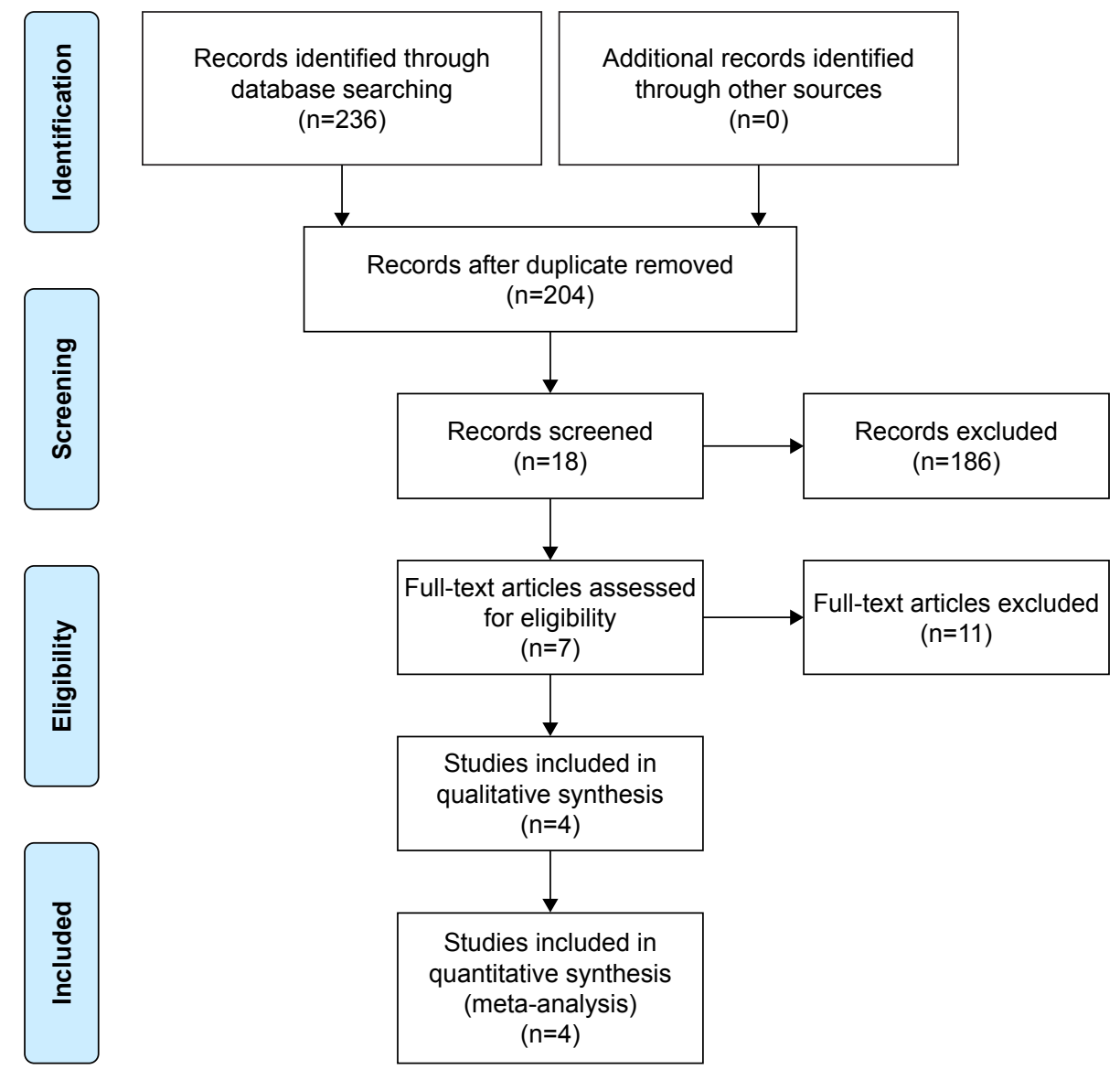

Figure I Flowchart of the study selection process. 


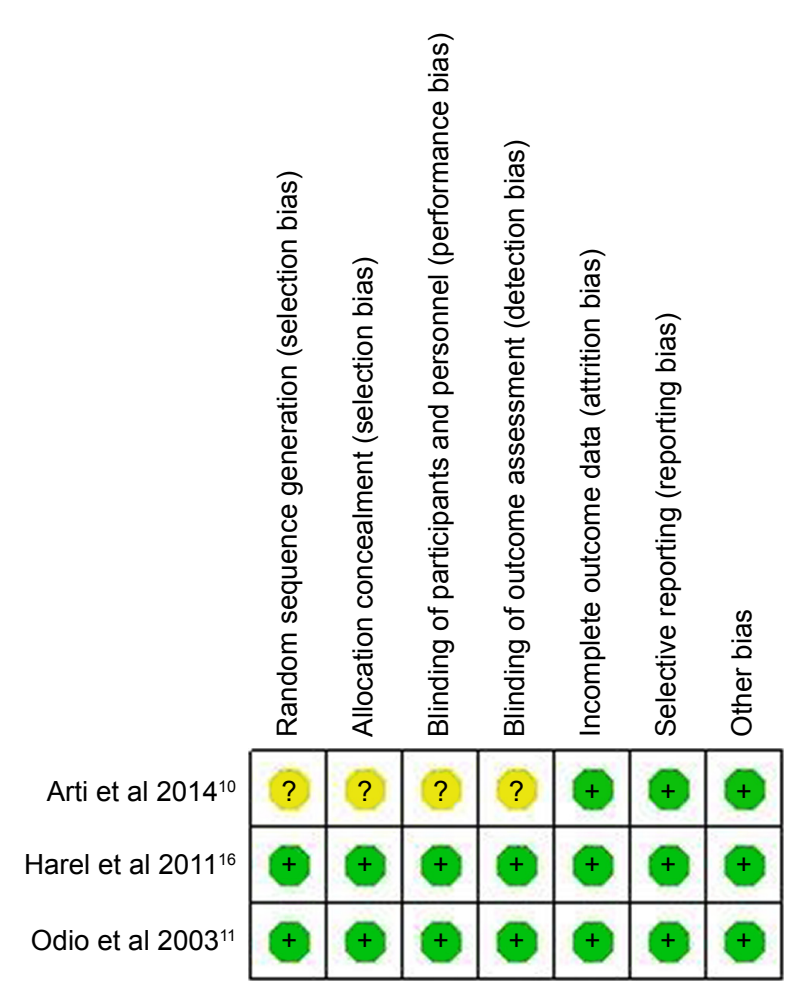

Figure 2 A risk of bias table for randomized controlled trials.

14 articles, because they did not compare dexamethasone group to placebo group in children with SA. Finally, four articles ${ }^{10,11,14,16}$ were included in our meta-analysis.

\section{Risk of bias assessment}

The scale of Cochrane Bone and the Joint and Muscle Trauma Group were used to evaluate the quality of the included studies of RCTs. The details are presented in Figure 2. The scores of one RCS evaluated by the MINORS quality assessment were 18. Table 2 summarizes more details of the quality assessment for RCS.

Table 2 Quality assessment score of the included studies

\begin{tabular}{|c|c|}
\hline Quality assessment for non-randomized trials & $\begin{array}{l}\text { Fogel et al } \\
2015^{14}\end{array}$ \\
\hline A clearly stated aim & 2 \\
\hline Inclusion of consecutive patients & 2 \\
\hline Prospective data collection & 0 \\
\hline Endpoints appropriate to the aim of the study & 2 \\
\hline Unbiased assessment of the study endpoint & 2 \\
\hline A follow-up period appropriate to the aims of study & 2 \\
\hline Less than $5 \%$ loss to follow-up & 0 \\
\hline Prospective calculation of the sample size & 0 \\
\hline An adequate control group & 2 \\
\hline Contemporary groups & 2 \\
\hline Baseline equivalence of groups & 2 \\
\hline Adequate statistical analyses & 2 \\
\hline
\end{tabular}

\section{Study characteristics}

Demographic characteristics and details concerning the literature type of the included studies are summarized in Table 3 . The studies included in the meta-analysis were from 2003 to 2015 and involved 348 patients (141 were treated with dexamethasone and 207 with placebo).

\section{Outcomes of meta-analysis}

\section{Number of days of hospitalization}

Two studies provided the number of days of hospitalization. A significant heterogeneity was found $\left(I^{2}=0.0 \%, P=0.524\right)$, and we used fixed-effect model. The results show 56 cases in the experimental and 120 in the control groups. The number of hospitalization days between the experimental group and the control group was statistically significant $(\mathrm{MD}=-4.226$, $95 \%$ CI: -4.785 to $-3.667, P=0.001$, Figure 3 ). The present meta-analysis shows that dexamethasone as adjunctive therapy with antibiotics can shorten the days of hospitalization in children with SA.

\section{Number of days to normalization of CRP}

Three studies provided the number of days to normalization of CRP. No obvious heterogeneity was observed, and a fixed model was used $\left(I^{2}=0.0 \%, P=0.728\right)$. The results show 111 cases in the experimental and 177 in the control groups. There was a significant difference in the number of days to normalization of CRP between the experimental and the control groups ( $\mathrm{MD}=-3.075,95 \% \mathrm{CI}:-3.362$ to -2.788 , $P=0.001$, Figure 4$)$. The present meta-analysis shows that the reduction of CRP in dexamethasone-treated patients was significantly faster than the reduction of CRP in patients in the placebo group.

\section{Number of days of intravenous antibiotic treatment}

Three studies provided the number of days of intravenous antibiotic treatment. We observed that the result did not show significant heterogeneity, and randomize-effect was used $\left(I^{2}=61.8 \%, P=0.073\right)$. The results show 111 cases in the experimental, and 177 in the control groups. There was a significant difference in the number of days of intravenous antibiotic treatment between the experimental and the control groups ( $\mathrm{MD}=-3.593,95 \% \mathrm{CI}:-4.825$ to $-2.361, P=0.001$, Figure 5). The present meta-analysis shows that dexamethasone as adjunctive therapy with antibiotics can shorten the number of days of treatment with intravenous antibiotics.

\section{Number of days of oral antibiotic treatment}

Two studies provided the number of days of oral antibiotics. No significant heterogeneity was found, and a fixed model 
Table 3 The characteristics of included studies

\begin{tabular}{|c|c|c|c|c|c|c|}
\hline \multirow[t]{2}{*}{ Study } & \multicolumn{2}{|c|}{ Sample size } & \multirow[t]{2}{*}{ Age } & \multirow[t]{2}{*}{ Glucocorticoid } & \multirow[t]{2}{*}{ Dose } & \multirow[t]{2}{*}{ Follow-up } \\
\hline & D & $\mathbf{P}$ & & & & \\
\hline Harel et al 201 ${ }^{16}$ & 24 & 25 & $34.74 * / 31.13 *$ & Dexamethasone & $0.15 \mathrm{mg} / \mathrm{kg}$ Q6 h 4 days & $12 *$ \\
\hline Odio et al $2003^{\prime \prime}$ & 61 & 62 & $5.55^{\ddagger} / 5.81^{\ddagger}$ & Dexamethasone & 0.20 mg/kg Q6 h 4 days & $12 *$ \\
\hline Arti et al $2014^{10}$ & 30 & 30 & $8.06^{\ddagger} / 8.00^{\ddagger}$ & Dexamethasone & $0.15 \mathrm{mg} / \mathrm{kg}$ Q6 h 4 days & NS \\
\hline Fogel et al $2015^{14}$ & 26 & 90 & $20.6 * / 27.4^{*}$ & Dexamethasone & $0.15 \mathrm{mg} / \mathrm{kg} \mathrm{Q} 6 \mathrm{~h} 4$ days & $12 *$ \\
\hline
\end{tabular}

Notes: *Months; ¥Year.

Abbreviations: $D$, dexamethasone; $P$, placebo; NS, not significant.

was used ( $\left.I^{2}=5.9 \%, P=0.303\right)$. The results show 85 cases in the experimental, and 87 in the control groups. There was a significant difference in the number of days of oral antibiotics between the experimental and the control groups $(\mathrm{MD}=-1.658,95 \% \mathrm{CI}:-2.539$ to $-0.777, P=0.001$, Figure 6 ). The present meta-analysis shows that adjunctive dexamethasone could shorten the number of days of oral antibiotic treatment.

\section{Discussion}

A vast array of pathogenic organisms has been identified as causing SA. The most common types of bacteria are the Grampositive cocci, such as Staphylococcus and Streptococcus. ${ }^{17,18}$ In fact, Kingella kingae, formerly called Moraxella kingae, is increasingly becoming an important pathogen of SA in patients $<3$ years of age. ${ }^{1}$ A recent study suggests that $82 \%$ of SA cases are caused by $K$. kingae in patients $<4$ years of age. ${ }^{19-21}$ Therefore, antibiotic application based on the organisms is the key to the treatment of SA. Although previous studies have demonstrated that adjunctive corticosteroids could improve the outcomes of severe sepsis, pneumonia, bacterial meningitis, and acute pyelonephritis, ${ }^{9,22-25}$ the efficacy and safety of adjunctive corticosteroids in SA were controversial. To our knowledge, this is the first quantitative meta-analysis to evaluate the efficacy of corticosteroids as adjunctive therapy with antibiotics in the treatment of children with SA.
Using plasma-CRP as an useful indicator of assessing anti-inflammatory therapeutic effect has been confirmed by other studies. ${ }^{26,27}$ The measurement of CRP as a useful index for assessing the response to SA treatment was initially performed in animal models. ${ }^{8}$ The first studies were conducted in humans in 2003. Odio et al ${ }^{11}$ observed that the CRP in dexamethasone-treated patients was reduced significantly faster than in control patients and reported that adjunctive dexamethasone could shorten the duration of symptoms and reduce residual dysfunction at the end of the treatment period and during follow-up. It is noteworthy that the results were similar to those reported recently by Harel et al. ${ }^{16}$ The present meta-analysis shows a shorter time to a decrease in CRP to normal levels in the dexamethasone group. Sakiniene et $\mathrm{al}^{8}$ concluded that the reason for the quicker drop of CRP could be explained by the inhibitory effect of the corticosteroids on T- and B-cell proliferation and differentiation, leading to a decrease in cytokine production.

The duration of antibiotic treatment and hospitalization was dependent on the clinical signs of improvement, absence of pathogenic bacteria of joint fluid, and a normal CRP. A consistent finding between the study by Fogel et $\mathrm{al}^{14}$ and our meta-analysis is a significant reduction in the duration of intravenous and oral antibiotic treatment. The children in the dexamethasone group experienced a significantly

\begin{tabular}{l|lc} 
Study ID & \multicolumn{2}{c}{ SMD (95\% Cl) } \\
\hline Arti et al $2014^{10}$ & $-4.48(-5.44$ to -3.52$)$ & 33.94 \\
Fogel et al $2015^{14}$ & $-4.10(-4.78$ to -3.41$)$ & \\
Overall $\left(I^{2}=0.0 \%, P=0.524\right)$ & $-4.23(-4.78$ to -3.67$)$ & \\
\hline-5.44 & & \\
\hline
\end{tabular}

Figure 3 Forest plot for meta-analysis of days of hospitalization. Abbreviation: SMD, standardized mean difference. 


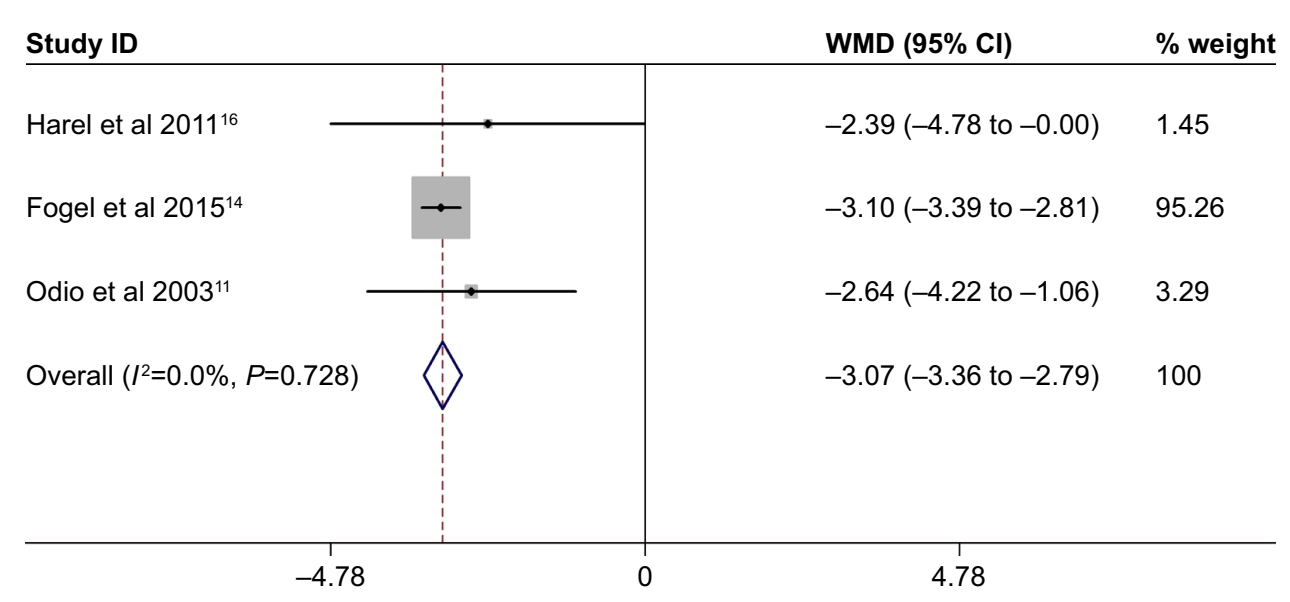

Figure 4 Forest plot for meta-analysis of days to normalization of C-reactive protein. Abbreviation: WMD, weight mean difference.

faster and more favorable clinical response than controls did and thus were switched to oral therapy at an earlier time. Furthermore, Fogel et $\mathrm{a}^{14}$ noted that the number of days of hospitalization was 8.0 and 10.7 days in the dexamethasone and placebo groups, respectively. Similar results were reported in other included studies and the present meta-analysis.

We were also concerned about the existence of adverse reactions, relapse, and sequelae since corticosteroids may hide the symptoms of infection and thereby lead to antibiotic treatment that is too short. Fogel et al ${ }^{14}$ reported four cases of relapse of transient symptoms less than a week after completing the corticosteroids course. At 12 months of follow-up, Odio et a ${ }^{11}{ }^{11}$ reported that 1 patient in the dexamethasone group had hip involvement with impaired angles of movement, limping, and shortening of the affected extremity, and 13 patients in the placebo group had dysfunction. Although no included study documented adverse events associated with corticosteroids, larger sample sizes and longer follow-up RCTs are further needed.

This study has the following limitations: 1) only three RCTs and one RCS were included in the present metaanalysis and the sample sizes were relatively small; 2) the included studies were short of some data, and we failed to perform a meta-analysis, such as functional score; and 3) follow-up was relatively short, which may lead to an underestimation of complications.

\section{Conclusion}

The present meta-analysis base points strongly toward a beneficial effect for corticosteroids in SA. Corticosteroids as adjunctive therapy with antibiotics in the treatment of children with SA could shorten the number of days of hospitalization, intravenous and oral antibiotics treatment, as well as the number of days to normalization of CRP. Therefore, we recommend corticosteroids as adjuvant therapy with antibiotics in the treatment of children with SA.

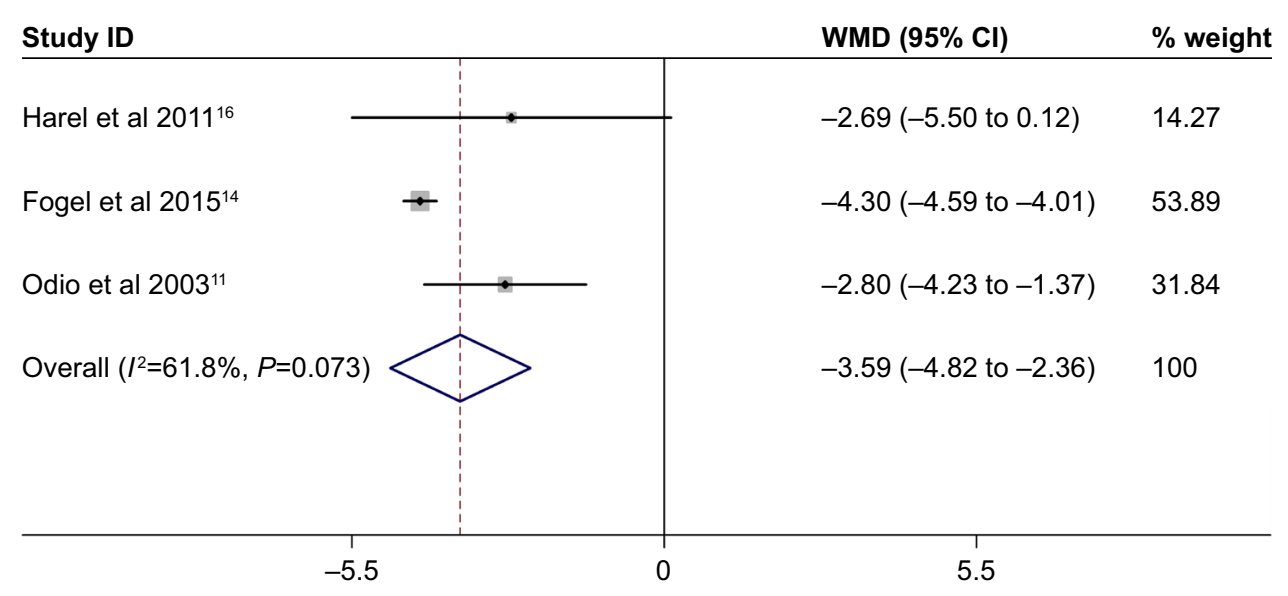

Figure 5 Forest plot for meta-analysis of days of intravenous antibiotics. Abbreviation: WMD, weight mean difference. 


\begin{tabular}{|c|c|c|}
\hline Study ID & WMD (95\% Cl) & $\%$ weight \\
\hline Harel et al $2011^{16}$ & $0.26(-3.49$ to 4.01$)$ & 5.52 \\
\hline Odio et al $2003^{11}$ & $-1.77(-2.68$ to -0.86$)$ & 94.48 \\
\hline Overall $\left(I^{2}=5.9 \%, P=0.303\right)$ & $-1.66(-2.54$ to -0.78$)$ & 100 \\
\hline
\end{tabular}

Figure 6 Forest plot for meta-analysis of days of oral antibiotics.

Abbreviation: WMD, weight mean difference.

\section{Acknowledgment}

This work was supported by fundings from National Natural Science Foundation of China (no 81501887).

\section{Disclosure}

The authors report no conflicts of interest in this work.

\section{References}

1. Agarwal A, Aggarwal AN. Bone and joint infections in children: septic arthritis. Indian J Pediatr. 2016;83(8):825-833.

2. Chiappini E, Mastrolia MV, Galli L, de Martino M, Lazzeri S. Septic arthritis in children in resource limited and non-resource limited countries: an update on diagnosis and treatment. Expert Rev Anti Infect Ther. 2016;14(11):1087-1096.

3. Howard JB, Highgenboten CL, Nelson JD. Residual effects of septic arthritis in infancy and childhood. JAMA. 1976;236(8):932-935.

4. Sáez-Llorens X, Mustafa MM, Ramilo O, Fink C, Beutler B, Nelson JD. Tumor necrosis factor alpha and interleukin 1 beta in synovial fluid of infants and children with suppurative arthritis. Am J Dis Child. 1990; 144(3):353-356.

5. Kwan Tat S, Padrines M, Théoleyre S, Heymann D, Fortun Y. IL-6, RANKL, TNF-alpha/IL-1: interrelations in bone resorption pathophysiology. Cytokine Growth Factor Rev. 2004;15(1):49-60.

6. Wysenbeek AJ, Volchek J, Amit M, Robinson D, Boldur I, Nevo Z. Treatment of staphylococcal septic arthritis in rabbits by systemic antibiotics and intra-articular corticosteroids. Ann Rheum Dis. 1998; 57(11):687-690.

7. Stricker SJ, Lozman PR, Makowski AL, Gunja-Smith Z. Chondroprotective effect of betamethasone in lapine pyogenic arthritis. $J$ Pediatr Orthop. 1996;16(2):231-236.

8. Sakiniene E, Bremell T, Tarkowski A. Addition of corticosteroids to antibiotic treatment ameliorates the course of experimental Staphylococcus aureus arthritis. Arthritis Rheum. 1996;39(9):1596-1605.

9. Buttgereit F, Burmester GR, Straub RH, Seibel MJ, Zhou H. Exogenous and endogenous glucocorticoids in rheumatic diseases. Arthritis Rheum. 2011;63(1):1-9.

10. Arti H, Mousapour A, Alavi SM. The effect of intravenous dexamethasone in the treatment of septic arthritis. Pak J Med Sci. 2014;30(5): 955-957.

11. Odio CM, Ramirez T, Arias G, et al. Double blind, randomized, placebocontrolled study of dexamethasone therapy for hematogenous septic arthritis in children. Pediatr Infect Dis J. 2003;22(10):883-889.

12. Narayanan UG. Dexamethasone added to antibiotics improved clinical and laboratory outcomes in children with septic arthritis. Bone Joint Surg Am. 2011;93(22):2124.
13. Macchiaiolo M, Buonuomo PS, Mennini M, Villani A, Bartuli A Question 2: Should steroids be used in the treatment of septic arthritis? Arch Dis Child. 2014;99(8):785-787.

14. Fogel I, Amir J, Bar-On E, Harel L. Dexamethasone therapy for septic arthritis in children. Pediatrics. 2015;136(4):e776-e782.

15. Farrow L. A systematic review and meta-analysis regarding the use of corticosteroids in septic arthritis. BMC Musculoskelet Disord. 2015; $16: 241$.

16. Harel L, Prais D, Bar-On E, et al. Dexamethasone therapy for septic arthritis in children: results of a randomized double-blind placebocontrolled study. J Pediatr Orthop. 2011;31(2):211-215.

17. Shirtliff ME, Mader JT. Acute septic arthritis. Clin Microbiol Rev. 2002;15(4):527-544.

18. Kaandorp CJ, Dinant HJ, van de Laar MA, Moens HJ, Prins AP, Dijkmans BA. Incidence and sources of native and prosthetic joint infection: a community based prospective survey. Ann Rheum Dis. 1997; 56(8):470-475.

19. Goldenberg DL. Septic arthritis. Lancet. 1998;351(9097):197-202.

20. Margaretten ME, Kohlwes J, Moore D, Bent S. Does this adult patient have septic arthritis? JAMA. 2007;297(13):1478-1488.

21. Till SH, Snaith ML. Assessment, investigation, and management of acute monoarthritis. J Accid Emerg Med. 1999;16(5): 355-361.

22. Colavite PM, Sartori A. Septic arthritis: immunopathogenesis, experimental models and therapy. J Venom Anim Toxins Incl Trop Dis. 2014;20:19.

23. Ceroni D, Cherkaoui A, Ferey S, Kaelin A, Schrenzel J. Kingella kingae osteoarticular infections in young children: clinical features and contribution of a new specific real-time PCR assay to the diagnosis. J Pediatr Orthop. 2010;30(3):301-304.

24. Brouwer MC, Heckenberg SG, de Gans J, Spanjaard L, Reitsma JB, van de Beek D. Nationwide implementation of adjunctive dexamethasone therapy for pneumococcal meningitis. Neurology. 2010;75(17): $1533-1539$.

25. de Gaudio M, Chiappini E, Galli L, de Martino M, Deg M, Dem M. Therapeutic management of bacterial meningitis in children: a systematic review and comparison of published guidelines from a European perspective. J Chemother. 2010;22(4):226-237.

26. Unkila-Kallio L, Kallio MJ, Peltola H. The usefulness of C-reactive protein levels in the identification of concurrent septic arthritis in children who have acute hematogenous osteomyelitis. A comparison with the usefulness of the erythrocyte sedimentation rate and the white blood-cell count. Bone Joint Surg Am. 1994;76(6): 848-853.

27. Kallio MJ, Unkila-Kallio L, Aalto K, Peltola H. Serum C-reactive protein, erythrocyte sedimentation rate and white blood cell count in septic arthritis of children. Pediatr Infect Dis J. 1997;16(4):411-413. 


\section{Publish your work in this journal}

Drug Design, Development and Therapy is an international, peerreviewed open-access journal that spans the spectrum of drug design and development through to clinical applications. Clinical outcomes, patient safety, and programs for the development and effective, safe, and sustained use of medicines are the features of the journal, which

has also been accepted for indexing on PubMed Central. The manuscript management system is completely online and includes a very quick and fair peer-review system, which is all easy to use. Visit http://www.dovepress.com/testimonials.php to read real quotes from published authors.

Submit your manuscript here: http://www.dovepress.com/drug-design-development-and-therapy-journal 